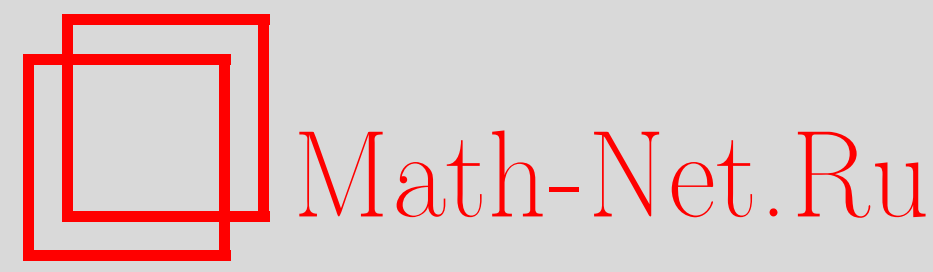

Э. Э. Шноль, Правильные многогранники и бифуркации симметричных положений равновесия обыкновенных дифференциальных уравнений, Матем. сб., 2000, том 191, номер 8, 141-157

DOI: https://doi.org/10.4213/sm503

Использование Общероссийского математического портала Math-Net.Ru подразумевает, что вы прочитали и согласны с пользовательским соглашением

http://www. mathnet.ru/rus/agreement

Параметры загрузки:

IP : 3.93.64.190

26 апреля 2023 г., 12:58:38 


\title{
Э.Э. Шноль \\ Правильные многогранники и бифуркации симметричных положений равновесия обыкновенных дифференциальных уравнений
}

\begin{abstract}
Рассматриваются все локальные однопараметрические бифуркации симметричных положений равновесия, отвечающие трехкратным нулевьм собственным значениям. В каждом случае соответствующая "бифуркационная группа" - ограничение полной группы симметрии дифференциальных уравнений на центральное многообразие - связана с симметрией правильного (трехмерного) многогранника. Показано, что во всех случаях, кроме одного, бифуркационные события исчерпьваются ветвлением положений равновесия. Доказательства основаны на существовании функций (типа функций Ллпунова), производная которых в силу уравнений сохраняет знак. Эти функции не зависят от бифуркационного параметра и имеют вид однородных функций нулевой степени.
\end{abstract}

Библиограб̆ия: 13 названий.

\section{§1. Постановка задачи и результаты}

1.1. Рассмотрим однопараметрическое семейство дифференциальных уравнений, инвариантное относительно действия конечной (или компактной) группы $\Gamma$ линейных преобразований:

$$
\begin{gathered}
\frac{d \mathbf{r}}{d t}=\mathbf{f}(\mathbf{r}, \alpha), \quad \mathbf{r}, \mathbf{f} \in \mathbb{R}^{n}, \quad \mathbf{f}(\mathbf{0}, \alpha) \equiv \mathbf{0} ; \\
\mathbf{f}(g \mathbf{r}, \alpha)=g \mathbf{f}(\mathbf{r}, \alpha) \quad \text { при } \quad g \in \Gamma .
\end{gathered}
$$

Для простоты мы предположили в (1.1), что $\mathbf{r}=\mathbf{0}$ есть положение равновесия при всех значениях параметра $\alpha$. Обший случай, когда положение равновесия $\mathbf{c}(\alpha)$ зависит от $\alpha$ и, возможно, инвариантно лишш относительно некоторой подгруппы $\Gamma$, легко сводится к этому, если группа Г конечна.

Если группа Г некоммутативна, то собственные значения $\lambda$, отвечающие равновесию $\mathbf{r}=\mathbf{0}$, могут быть кратными при всех значениях параметра $\alpha$. Чем выше кратность $\nu$, тем сложнее могут быть бифуркации этого положения равновесия. Причина очевидна: размерность модельных систем, отвечающих за бифуркационные события, равна $\nu$ для вешественных $\lambda$ и равна $2 \nu$ для мнимых $\lambda$. Для двукратных нулевых $\lambda$ все просто: никаких бифуркационных событий, кроме ветвления положений равновесия, здесь не происходит. Для двукратных мнимых $\lambda$ изучены не все возможные бифуркации. Здесь при изменении одного параметра из равновесия может рождаться двумерный тор (с фиксированным числом циклов на нем) или инвариантная для уравнений трехмерная сфера [1].

Работа вьполнена при финнансовой поддержке фонда INTAS (грант № 93-893).

$$
\text { (C) Э. Э. Шноль } 2000
$$


В настоящей статье обсуждаются все локальные (в смысле [2]) бифуркации симметричных положений равновесия, отвечающие коразмерности 1 и трехкратному нулевому $\lambda$. Общий итог прост: как и при кратности два, здесь не происходит никаких бифуркационных событий, кроме ветвления равновесий. Более точно, во всех случаях, кроме одного, справедливо следующее утверждение. Существует окрестность точки $\mathbf{r}=\mathbf{0}$ такая, что любая траектория системы для значений параметра $\alpha$, близких к бифуркационному, стремится к одному из положений равновесия или покидает эту окрестность (не зависяшую от $\alpha$ ). В особом случае в роли аттрактора может выступать еше цикл из сепаратрис седловых равновесий. Таким образом, здесь симметрия "запрещает” сколько-нибудь сложное поведение решений, возникающих при бифуркации (a priori возможное в трехмерных системах).

Подчеркнем лишний раз (см. [3; раздел $32 \mathrm{~A}]$ ), что в упомянутых простейших случаях, так же как в общей, "несимметричной” теории бифуркаций, желательно дать достаточно полное описание локально фазового портрета в области, не зависящей от параметра.

Несколько слов о месте рассматриваемой задачи в теории бифуркаций симметричных дифференциальных уравнений. При общем рассмотрении, пока не фиксирована группа симметрии Г (или класс изучаемых уравнений), кажется правильным рассмотреть все бифуркационные задачи до некоторого уровня сложности. Кратность $\nu=3$ для нулевых $\lambda$ и кратность $\nu=2$ для чисто мнимых $\lambda$ суть естественные границы для такого рода исследований однопараметрических бифуркаций по двум причинам.

Во-первых, набор различных задач при большей кратности плохо обозрим. Так, для трехкратных мнимых $\lambda$ роль “бифуркационной группы" $G$ (см. п. 1.2) может играть любая подгруппа группы унитарных матриц U(3). Во-вторых, сколько-нибудь полное описание фазового портрета, видимо, возможно здесь лишь в редких случаях. Имеются, в частности, замечательные примеры, показывающие, что из равновесия могут рождаться (в качестве бифуркаций коразмерности 1) малые “хаотические” колебания. Для этого достаточно появления трехкратной пары чисто мнимых $\lambda[4]$ или нулевого $\lambda$ кратности 4 [5].

1.2. Пусть $L_{\alpha}=\mathbf{f}_{\mathbf{r}}^{\prime}(\mathbf{0}, \alpha)$ - оператор линеаризации в положении равновесия $\mathbf{r}=\mathbf{0}$. Операторы $L_{\alpha}$ коммутируют с преобразованиями $g$ из Г. Без ограничения общности можно считать, что элементы группы $\Gamma$ - ортогональные преобразования в смысле некого скалярного произведения. Возможная кратность собственных значений $L_{\alpha}$ при заданной групше $\Gamma$ определяется разложением фазового пространства на минимальные инвариантные для Г подпространства. В частности, трехкратное нулевое $\lambda$ появляется в типичном семействе операторов $L_{\alpha}$, если в таком разложении есть (хотя бы одно) трехмерное подпространство $E$ [6; гл. 13$]$. Вблизи бифуркационного значения параметра $\alpha$, которое мы будем полагать равньп нулю, подпространство $E$ состоит из собственных векторов $L_{\alpha}$, отвечающих собственному значению $\lambda(\alpha)(\lambda(0)=0)$ - жордановы клетки здесь не появляются. Ограничение действия $\Gamma$ на подпространство $E$ дает неприводимое представление $\Gamma \mapsto G$. В нашем случае $G$ есть подгруппа группы ортогональных преобразований $\mathrm{O}(3)$ трехмерного пространства, действующая в нем неприводимо (не имеющая инвариантных двумерных подпространств). Возможности таковы $[7$; раздел 13], [8; раздел 93]: $G$ совпадает с $\mathrm{O}(3)$ (или $\mathrm{SO}(3))$ либо изоморфна одной из семи конечных груп, связанных с правильными многогранниками. 
Трехмерное центральное многообразие $\mathbf{M}_{\alpha}$ системы (1.1) (определенное при малых $\alpha$ ) касается подпространства $E$. Его можно считать инвариантным относительно действия группы Г (см., например, [9]) и использовать декартовы координаты на $E$ в качестве координат на $\mathbf{M}_{\alpha}$.

Бифуркационные события определяются тем, что происходит на $\mathbf{M}_{\alpha}$, и мы приходим к следующей задаче: изучить бифуркации коразмерности 1 в системе трех уравнений $\dot{\mathbf{r}}=\mathbf{f}(\mathbf{r}, \alpha)$, инвариантной относительно группь $G \subset \mathrm{O}(3)$, которая действует в $\mathbb{R}^{3}$ неприводимо. Здесь векторноеполе $\mathbf{f}(\mathbf{r}, \alpha)=\lambda(\alpha) \mathbf{r}+\cdots$ $G$-эквивариантно, т.е. удовлетворяет условию (1.2) для всех $g \in G$.

Случай, когда система обладает полной сферической симметрией, тривиален. Остаются следуюшие случаи, которые и будут предметом рассмотрения.

1. $G$ изоморфна группе $T$ вращений правильного тетраэдра (из 12 элементов) или полной группе его симметрий, включающей отражения в плоскостях симметрии.

2. $G$ изоморфна группе $O$ врашений куба или полной группе его симметрий (включающей отражения).

3. $G$ изоморфна группе $Y$ врашений додекаэдра (или икосаэдра) либо полной группе его симметрий.

\section{4. $G$ порождается $T$ и центральной симметрией (содержит 24 элемента).}

В случаях 1, 2 и 3 инвариантность относительно отражений (увеличение группы симметрии) не привносит существенных изменений. Все рождающиеся при бифуркации положения равновесия находятся на осях симметрии соответствуюшего правильного многогранника.

Отметим, что в случаях 1-3 нелинейные слагаемые наименьшей степени образуют градиентное векторное поле. Это и есть в конечном счете причина простоты бифуркационной картины. Однако в случае 1 нужно учесть кроме квадратичных еще и кубические члены, которые (при групе $T$ ) не градиентны. Соответственно здесь возникают две серии положений равновесия: отстоящие от $\mathbf{r}=\mathbf{0}$ на расстояния порядка $|\alpha|$ и порядка $|\alpha|^{1 / 2}$. Случай 2 самый простой. Здесь квадратичных членов нет, а кубичные образуют (вместе с линейными) градиентную систему, которая все и определяет. В случае 3 линейные и кубичные члены дают систему, обладающую полной сферической симметрией. Дискретность группы симметрии проявляется лиш в членах пятой степени, которые, следовательно, необходимо учитывать.

Доказательства для всех случаев основаны на существовании “функции Ляпунова" (в виде однородной функции нулевой степени, не зависящей от параметра). В $\S \S 2$ и 4 подробно рассмотрен случай тетраэдральной симметрии, в $\S 5$ - случай кубической симметрии и в $\S 6$ - случай симметрии додекаэдра (где несколько сложнее алгебра). Случай 4 кратко рассмотрен в $\S 7$. Алгебраические рассмотрения, играющие в нашей теме вспомогательную роль, вынесены в дополнение.

Автор благодарен Э.Б. Винбергу за разъяснения, касающиеся инвариантов групп симметрии правильных многогранников, и соответствуюшие литературные указания. 


\section{§2. Тетраэдральная симметрия (начало)}

2.1. Выбор декартовых координат. Пусть $T \subset \mathrm{SO}(3)$ - группа вращений правильного тетраэдра. Выберем оси координат так, чтобы они были осями симметрии второго порядка (т.е. чтобы в группу $T$ входили повороты на $180^{\circ}$ вокруг этих осей). При этом четыре биссектрисы координатных октантов $(x=y=z$ и т.д.) будут осями симметрии третьего порядка.

Будем обозначать через $\mathbf{f}_{n}=\left(u_{n}, v_{n}, w_{n}\right)$ векторные поля, компоненты которых - однородные многочлены степени $n$.

ЛЕмма 2.1. а) T-эквивариантное векторное поле $\mathbf{f}_{2}$ имеет вид

$$
u_{2}=q y z, \quad v_{2}=q z x, \quad w_{2}=q x y ;
$$

б) $T$-эквивариантное векторное поле $\mathbf{f}_{3}$ имеет вид

$$
u_{3}=x\left(a x^{2}+b y^{2}+c z^{2}\right), \quad v_{3}=y\left(a y^{2}+b z^{2}+c x^{2}\right), \quad w_{3}=z\left(a z^{2}+b x^{2}+c y^{2}\right) .
$$

Здесь $q, a, b, c-$ произвольные постояннье.

\section{ДокАЗАТЕЛЬСТво. Напишем}

$$
u_{3}=a x^{3}+x^{2} P_{1}(y, z)+x P_{2}(y, z)+P_{3}(y, z) .
$$

При повороте вокруг оси $x$ на $180^{\circ} x \rightarrow x, y \rightarrow-y, z \rightarrow-z$ и должно быть $u \rightarrow u$. Отсюда $P_{1}=P_{3} \equiv 0$. При повороте вокруг оси $y$ на $180^{\circ}$ должно быть $u \rightarrow-u$. Отсюда $P_{2}=b y^{2}+c z^{2}$. При повороте вокруг оси $x=y=z$ на $120^{\circ} u \rightarrow v$ и $v \rightarrow w$. Поэтому соответствующие коэффициенты в формулах (2.2) равны.

У тверждение а) доказывается аналогично.

ЗАмечАния. 1) В полную группу $T_{d}$ симметрий тетраэдра входят отражения в плоскостях $x= \pm y, y= \pm z$ и $z= \pm x$ (порождающие эту группу). Векторное поле (2.1) эквивариантно относительно $T_{d}$. Поле (2.2) $T_{d}$-эквивариантно только при $b=c$.

2) Векторное поле (2.1) градиентно - порождается инвариантом $I_{3}=q x y z$. Кубичное векторное поле (2.2) градиентно при $b=c$.

Оба эти факта можно предвидеть без вычислений (см. дополнение).

2.2. Укороченная система. Пусть система дифференциальных уравнений $\dot{\mathbf{r}}=\mathbf{f}(\mathbf{r})$ в $\mathbb{R}^{3}(\cdot=d / d t)$ инвариантна относительно группы $T$, действуюшей как описано вьше. Прямые, являющиеся вращательными осями группы симметрии (как и для случаев других подгрупп $\mathrm{SO}(3)$ ), инвариантны для рассматриваемой системы. Ограничившись линейньми и квадратичными членами, получим следующие уравнения

$$
\dot{x}=\gamma x+q y z, \quad \dot{y}=\gamma y+q z x, \quad \dot{z}=\gamma z+q x y .
$$

Лучи, составляющие 7 осей симметрии групшы $T$, инвариантны для системы (2.3). При $q$, отличном от нуля, других инвариантных лучей у нее нет. Система (2.3) градиентная, ее траектории при $t \rightarrow \pm \infty$ стремятся к положениям равновесия или уходят на бесконечность. 
Введем сферические координаты. Более точно, для каждого $\mathbf{r}$ положим $R=$ $|\mathbf{r}|=\left(x^{2}+y^{2}+z^{2}\right)^{1 / 2}$ и $\Omega=\mathbf{r} / R$. Далее, положим $d \tau=R d t$. Не фиксируя координаты на единичной сфере $S^{2}$, которую пробегает $\Omega$, перепишем систему (2.3) (штрих заменяет $d / d \tau$ ):

$$
\begin{aligned}
& R^{\prime}=\gamma+q R h(\Omega) ; \\
& \Omega^{\prime}=\Phi(\Omega) \equiv q H(\Omega)
\end{aligned}
$$

( $h$ и $H$ не содержат $q$ и $\gamma)$. Двумерная "угловая" подсистема $(2.4 \mathrm{~b})$ имеет на сфеpe $S^{2}$ (при $q \neq 0$ ) 14 положений равновесия $\Omega_{k}$, отвечающих инвариантным лучам системы (2.3). Вводя локальные координаты, легко показать, что восемь из них узловые, а шесть, отвечающих координатным осям, седловые. Соответствующие собственные значения отличны от нуля (пропорциональны $q$ ).

2.3. Градиентность "угловой" системы. Любая траектория системы (2.4b), отличная от положения равновесия, при $\tau \rightarrow+\infty$ и при $\tau \rightarrow-\infty$ стремится к одному из равновесий. В этом можно убедиться, заметив, что система (2.4b) не зависит от $\gamma$, а при $\gamma=0$ поведение траекторий однородной системы $(2.3)$ нетрудно исследовать. Вместо этого мы используем то, что система $(2.4 \mathrm{~b})$ на двумерной сфере градиентна ${ }^{1}$, основываясь на следующей лемме (полезной и в других случаях).

2.4. ЛЕмма 2.2. Пусть задана однородная система $\dot{\mathbf{r}}=\mathbf{q}(\mathbf{r})$ в евклидовом пространстве $\mathbb{R}^{n}$ (любой размерности). Предположсим, что она градиентна - порождается функиией $I(R, \Omega)=R^{s+1} J(\Omega)(R=|\mathbf{r}|, \Omega=\mathbf{r} / R)$. Тогда соответствующая угловая система $\Omega^{\prime}=\Phi(\Omega)$ также градиентна - порождается функиией $J(\Omega)\left(\right.$ итрих $\left.=d / d \tau, d \tau=R^{s-1} d t\right)$.

ДокАЗАтельство. В пространстве $\mathbb{R}^{n}$ запишем $d s^{2}=d R^{2}+R^{2} d \Omega^{2}$. Пусть $\xi^{i}$ - локальные координаты на единичной сфере и $d \Omega^{2}=\widetilde{g}_{i k} d \xi^{i} d \xi^{k}(i, k=1, \ldots, n-1)$. В переменных $R, \xi$ из системы $\dot{\mathbf{r}}=\mathbf{q}(\mathbf{r})$ следует

$$
\frac{d \xi^{i}}{d t}=\frac{1}{R^{2}} \widetilde{g}^{i k} \frac{\partial I}{\partial \xi^{k}}=R^{s-1} \widetilde{g}^{i k} \frac{\partial J}{\partial \xi^{k}} .
$$

Положив $d \tau=R^{s-1} d t$, получим из (2.5) утверждение леммы.

В нашем случае $s=2, J(\Omega)=q x y z / R^{3}$. В стандартных сферических координатах: $4 J(\theta, \varphi)=q \cos \theta \cdot \sin 2 \theta \cdot \sin 2 \varphi$. На каждой траектории системы $(2.4 \mathrm{~b})$, отличной от положения равновесия, функция $J$ монотонно возрастает.

2.5. Используем теперь переменные $R, \Omega, \tau$ для “полной" системы $\dot{\mathbf{r}}=\mathbf{f}(\mathbf{r})$. Вместо (2.4) мы получим

$$
\begin{gathered}
R^{\prime}=\gamma+q R h(\Omega)+h_{1}(R, \Omega), \quad h_{1}=O\left(R^{2}\right) \\
\Omega^{\prime}=\Phi(\Omega)+\Psi(R, \Omega), \quad \Psi=O(R) .
\end{gathered}
$$

\footnotetext{
${ }^{1}$ Векторное поле $A$ на римановом многообразии градиентно, если в локалњных координа$\operatorname{tax}\left\{\xi^{i}\right\}$ его компоненты (в стандартных обозначениях) имеют вид $A^{i}=g^{i k} \partial F / \partial \xi^{k}$. Здесь $F$ - некая скалярная функция.
} 
Лучи $\Omega=\Omega_{k}\left(\Phi\left(\Omega_{k}\right)=0\right)$ инвариантны для полной системы и, следовательно, $\Psi\left(R, \Omega_{k}\right) \equiv 0$. Рассмотрим решение системы $(2.6)$, для которого $R(\tau) \leqslant R_{*}$ при всех $\tau \geqslant 0$. Если $R_{*}$ достаточно мало, то второе слагаемое в $(2.6 \mathrm{~b})$ можно рассматривать как малое возмущение. Считая $R(\tau)$ заданным, будем рассматривать (2.6b) как неавтономное уравнение, мало отличающееся от градиентной автономной системы $\Omega^{\prime}=\Phi(\Omega)$. При этом возмущение специфично: оно сохраняет все положения равновесия невозмушенной системы.

\section{§3. Неавтономное возмушение градиентной системы}

Рассмотрим на компактном многообразии с заданной римановой метрикой две системы дифференциальных уравнений:

$$
\begin{aligned}
& \frac{d \xi}{d t}=\Phi(\xi), \\
& \frac{d \xi}{d t}=\Phi(\xi)+\Psi(\xi, t) .
\end{aligned}
$$

Предположим, что "невозмушенная" система (3.1) градиентна - порождается функцией $J(\xi)$. Предположим, далее, что эта система имеет конечное число положений равновесия $s_{k}$ и что все они гиперболичны (не имеют нулевых собственных значений).

3.1. ЛЕмма 3.1. Пусть система (3.1) обладает описанными свойствами, а функиия $\Psi(\xi, t)$ такова, что $\Psi\left(s_{k}, t\right) \equiv 0: s_{k}$ являются положсениями равновесия и для системы (3.2). Пусть при всех $\xi u t \geqslant t_{0}$

$$
\begin{gathered}
\|\Psi\| \leqslant m, \\
\left\|\frac{\partial \Psi}{\partial \xi}\right\| \leqslant m .^{2}
\end{gathered}
$$

Если $m \leqslant m_{*}$, то каждое решение $\xi(t)$ системь (3.2) при $t \rightarrow \infty$ стремится $к$ одному из положсений равновесия $s_{k}\left(\right.$ либо $\left.\xi(t) \equiv s_{k}\right)$.

ДокАЗАТЕЛЬСТво. Производная функции $J$ в силу системы $(3.1)^{3}$ равна $\|\operatorname{grad} J\|^{2}$. Она неотрицательна и равна нулю лишь в точках $s_{k}$. Покажем, что при малых $m$ это свойство сохраняется для системы (3.2).

1) По условию критические точки $s_{k}$ функции $J$ невырождены. Отсюда следует, что у каждой точки $s_{k}$ есть окрестность $U_{k}$ такая, что для $\xi \in U_{k} c \rho\left(\xi, s_{k}\right) \leqslant$

\footnotetext{
${ }^{2}$ Нормы векторов и ковекторов определены стандартно - с помощью метрического тензоpa $g_{i k}$. Норма тензора $\partial \Psi / \partial \xi$ определена как норма линейного оператора, действующего в касательном пространстве.

${ }^{3}$ Пусть на многообразии заданы скалярная функция $F(\xi)$ и диффференциальное уравнение $\dot{\xi}=v(\xi, t)$. Производная $F$ в силу уравнения есть по определению функция $F_{d}$, задаваемая в (локальных) координатах формулой $F_{d}(\xi, t)=\sum v_{k}(\xi, t) \partial F / \partial \xi^{k}$. Если $\xi=\varphi(t)$ - произвольное решение дифференциального уравнения, то $\dot{F}(\varphi(t))=F_{d}(\varphi(t), t)$.
} 
$\|\operatorname{grad} J\| \leqslant C \rho\left(\xi, s_{k}\right)$. Здесь $\rho-($ кратчайшее) расстояние между точками. Поскольку $\Psi\left(s_{k}, t\right)=0$, то в силу (3.3b) имеем в $U_{k}:\|\Psi(\xi, t)\| \leqslant m \rho\left(\xi, s_{k}\right)$. Производная $J$ в силу системы $(3.2)$ есть $J_{d}(\xi, t)=\|\operatorname{grad} J\|^{2}+(\operatorname{grad} J, \Psi)$. Имеем

$$
J_{d} \geqslant\|\operatorname{grad} J\|^{2}-\|\operatorname{grad} J\| \cdot\|\Psi\| \text {. }
$$

В окрестности $U_{k}$ получим

$$
J_{d}(\xi, t) \geqslant\left(c^{2}-C m\right) \rho^{2}\left(\xi, s_{k}\right)
$$

2) Вне всех окрестностей $U_{j}$ выполнены неравенства $C_{1} \leqslant\|\operatorname{grad} J\| \leqslant C$ $\left(C_{1}>0\right)$. Из (3.4) и (3.3a) следует, что здесь $J_{d} \geqslant C_{1}^{2}-C m$. Отсюда и из (3.5) вытекает, что функция $J_{d}(\xi, t)$ неотрицательна при $m \leqslant m_{*}$, если $m_{*}$ меньше, чем $c^{2} / C$ и $C_{1}^{2} / C ; J_{d}(\xi, t)$ обрашается в нуль только при $\xi=s_{k}$.

3 ) Пусть $\xi(t)$ - некоторое решение системы (3.2), отличное от всех равновесий $s_{k}$. Функция $V(t)=J(\xi(t))$ с ростом $t$ возрастает: производная $\dot{V}(t)=J_{d}(\xi(t), t)$ положительна. Легко проверить, что вторая производная $\ddot{V}(t)$ ограничена. Отсюда $\dot{V}(t) \rightarrow 0$ при $t \rightarrow \infty$, т.е. $\xi(t)$ стремится к одному из положений равновесия $s_{k}$.

Добавление. Предположим, что системы (3.1), (3.2) гладко зависят от параметра $\alpha$ и при каждом $\alpha$ из некоторого отрезка выполняются предположения леммы 3.1. Тогда можно выбрать $m_{*}$ не зависящим от $\alpha$.

\section{§4. Тетраэдральная симметрия (окончание)}

4.1. ТЕОРЕМА 4.1. Пусть семейство дифференциальных уравнений $\dot{\mathbf{r}}=$ $\mathbf{f}(\mathbf{r}, \alpha)$ в $\mathbb{R}^{3}$ инвариантно относительно группь вращений тетраэдра $T$ или полной группьи его симметрий. Тогда

$\{1\}$ в подходящих декартовых координатах уравнения имеют вид

$$
\begin{aligned}
& \dot{x}=\lambda(\alpha) x+q y z+x\left(a x^{2}+b y^{2}+c z^{2}\right)+O\left(R^{4}\right), \\
& \dot{y}=\lambda(\alpha) y+q z x+y\left(a y^{2}+b z^{2}+c x^{2}\right)+O\left(R^{4}\right), \\
& \dot{z}=\lambda(\alpha) z+q x y+z\left(a z^{2}+b x^{2}+c y^{2}\right)+O\left(R^{4}\right) .
\end{aligned}
$$

Здесь $R^{2}=x^{2}+y^{2}+z^{2}$ u $q, a, b$, с есть функиии от $\alpha$.

Предположим, что $\lambda(0)=0$ и выполнены следующие условия невырожсденности:

$$
\lambda_{1}=\lambda^{\prime}(0) \neq 0, \quad q_{0}=q(0) \neq 0, \quad a_{0}=a(0) \neq 0 .
$$

Тогда при достаточно мальх $\alpha\left(|\alpha|<\varepsilon_{*}\right)$ существует шар $B\left(R \leqslant R_{*}\right)$ со следующими свойствами:

$\{2\}$ если $\alpha \neq 0$, то на каждой из 4 прямых $x= \pm y= \pm z$ в $B$ имеется ровно одно положсение равновесия системы, отличное от $\mathbf{r}=\mathbf{0}$;

$\{3\}$ если $\alpha \neq 0$ u $\operatorname{sign} \alpha=-\operatorname{sign}\left(\lambda_{1} / a_{0}\right)$, то на каждой из координатных осей в В имеется ровно два положсения равновесия системь, отличныз от $\mathbf{r}=\mathbf{0}$

$\{4\}$ каждая полутраектория системь, начинающаяся в $B$, с ростом $t$ либо стремится $\kappa \mathbf{r}=\mathbf{0}$ или одному из указанных положсений равновесия, либо покидает иар $B$ за конечное время. 
ДоказАтЕльство. 1) Справедливость утверждения $\{1\}$ следует из п. 2.1.

2) Утверждения $\{2\}$ и $\{3\}$ вытекают из инвариантности осей симметрии для системы (4.1): на каждой из этих прямых система сводится к одному уравнению. На оси $x=y=z$ и других осях третьего порядка получим уравнение $\dot{x}=\lambda_{1} \alpha x+$ $q_{0} x^{2}+O\left(\alpha^{2}|x|+|\alpha| x^{2}+|x|^{3}\right)$. Его правая часть обрашается в нуль при $x=0$ и при $x=-\lambda_{1} \alpha / q_{0}+O\left(\alpha^{2}\right)$. При $|\alpha| \leqslant \varepsilon_{1}$ и $R \leqslant R_{1}$ других положений равновесия уравнение не имеет. На оси $x$ система сводится к уравнению вида $\dot{x}=\lambda_{1} \alpha x+$ $a_{0} x^{3}+O\left(\alpha^{2}|x|+\left|\alpha x^{3}\right|+x^{4}\right)$. Его правая часть обрашается в нуль при $x=0$ и при $x= \pm\left(-\lambda_{1} \alpha / a_{0}\right)^{1 / 2}+O(|\alpha|)$. При $|\alpha| \leqslant \varepsilon_{2}$ и $R \leqslant R_{2}$ других положений равновесия это уравнение не имеет. $\mathrm{K}$ аналогичному уравнению сводится система на координатных осях $y$ и $z$.

3) Перейдем к доказательству основного утверждения $\{4\}$. Записав систему (4.1) в переменных $R, \Omega, \tau$ (см. п. 2.2), получим для $\Omega$ уравнение вида $(2.6 \mathrm{~b}):$

$$
\begin{aligned}
& \Omega^{\prime}=q(\alpha) H(\Omega)+\Psi(R, \Omega, \alpha), \\
& \|\Psi\| \leqslant C R, \quad\left\|\frac{\partial \Psi}{\partial \Omega}\right\| \leqslant C R .
\end{aligned}
$$

Здесь векторное поле $H(\Omega)$ градиентно - порождается функцией $J_{1}(\Omega)=x y z / R^{3}$. Пусть $q(\alpha) \neq 0$ на отрезке $|\alpha| \leqslant \varepsilon_{0}$ (см. (4.2)). Фиксируем произвольное $\alpha$ из этого отрезка и рассмотрим некоторое решение $\mathbf{r}(t)$ системы (4.1) (отличное от $\mathbf{r}(t) \equiv \mathbf{0}$ ). Подставив в (4.3) функцию $R(\tau)$, получим неавтономную систему, решением которой является $\Omega(\tau)$. Эта система принадлежит к тому типу, к которому применима лемма 3.1. Пусть $m_{*}$ задает верхнюю границу возмушения, при котором справедливо утверждение леммы (величина $m_{*}$ определяется первым слагаемым в правой части (4.3)). Предположим, что $R(\tau) \leqslant R_{0}$ при всех $\tau \geqslant 0$. Выберем $R_{0}=m_{*} / C$ (см. (4.4)). Тогда в силу указанной леммы $\Omega(\tau)$ при $\tau \rightarrow \infty$ стремится к одному из положений равновесия системы $\Omega^{\prime}=H(\Omega)$.

4) Предположим, что $\mathbf{r}(t)$ не стремится к точке $\mathbf{r}=\mathbf{0}$ при $t \rightarrow \infty$. Тогда интервалу $t_{0}<t<\infty$ отвечает для этого решения интервал $0<\tau<\infty$, поскольку интеграл от $R(t)$ расходится (напомним, что $R(t) d t=d \tau$ и что $\dot{R}(t)$ ограничено). Итак, решение $\mathbf{r}(t)$, оставаясь в шаре $\|\mathbf{r}\| \leqslant R_{0}$, при $t \rightarrow \infty$ неограниченно приближается к одному из инвариантных лучей системы (4.1). Следовательно, $\mathbf{r}(t)$ при $t \rightarrow \infty$ стремится к положению равновесия (отличному от $\mathbf{r}=\mathbf{0}$ ), находящемуся на этом луче.

Утверждение $\{4\}$ будет справедливо, если выбрать $R_{*}=\min \left(R_{0}, R_{1}, R_{2}\right)$ и $\varepsilon_{*} \leqslant \min \left(\varepsilon_{0}, \varepsilon_{1}, \varepsilon_{2}\right)$ ( $\varepsilon_{*}$ должно быть достаточно мало, чтобы указанные вьше равновесия попадали в шар $\left.R \leqslant R_{*}\right)$.

\section{§5. Кубическая симметрия}

5.1. Выбор декартовых координат. Пусть $O \subset \mathrm{SO}(3)$ - группа врашений куба. Выберем оси координат параллельными ребрам куба, а начало координат в его центре. Оси координат будут осями симметрии четвертого порядка, а биссектрисы координатных октантов ( $x=y=z$ и т.д.) будут осями третьего порядка. 
Кроме того, есть 6 поворотных осей второго порядка - биссектрисы квадрантов в каждой из координатных плоскостей (линии $z=0, x=y$ и т. д.).

Поскольку $O \supset T$, справедлива лемма 2.1. Дополнительные элементы симметрии позволяют усилить утверждения этой леммы. Именно:

a) $O$-эквивариантное векторное поле $\mathbf{f}_{2}$ тождественно равно нулю;

б) O-эквивариантное векторное поле $\mathbf{f}_{3}$ имеет вид $(2.2)$ с $c=b$.

Действительно, при повороте вокруг оси $x$ на $90^{\circ} y \rightarrow z, z \rightarrow-y$. При этом $u_{2}=q y z$ и $u_{3}=x\left(a x^{2}+b y^{2}+c z^{2}\right)$ не должны меняться. Отсюда $q=0$ и $b=c$.

5.2. Инвариантные системы. Пусть система дифференциальных уравнений $\dot{\mathbf{r}}=\mathbf{f}(\mathbf{r})$ в $\mathbb{R}^{3}(\cdot=d / d t)$ инвариантна относительно групшы $O$, действующей как описано выше. В силу п. 5.1 она имеет вид $\dot{\mathbf{r}}=\gamma \mathbf{r}+\mathbf{f}_{3}(\mathbf{r})+O\left(R^{4}\right)$. Тринадцать вращательных осей группы $O$ инвариантны для системы; на каждой из них система сводится к уравнению вида $\dot{s}=\gamma s+A s^{3}+O\left(s^{4}\right)(s-$ одна из координат). Здесь $A=a$ для осей четвертого порядка, $A=a+2 b$ для осей третьего порядка и $A=a+b$ для осей второго порядка.

Введем, как в п. 2.2, переменные $R$ и $\Omega$ ( $\Omega$ пробегает единичную сферу) и положим $d \tau=R^{2} d t$. Отбросив в системе слагаемые $O\left(R^{4}\right)$, получим градиентную “угловую” систему $\Omega^{\prime}=\Phi(\Omega)$ (см. п. 2.4). Лучам, инвариантным для системы $\dot{\mathbf{r}}=\gamma \mathbf{r}+\mathbf{f}_{3}(\mathbf{r})$, отвечают положения равновесия $\Omega_{k}$ угловой системы и наоборот. Легко проверить, что при $a \neq b$ инвариантны только лучи, составляющие оси симметрии куба. Им отвечают 26 гиперболических положений равновесия $\Omega_{k}$.

5.3. ПРЕДЛОЖЕНИЕ 5.1. Пусть семейство дифференииальных уравнений $\dot{\mathbf{r}}=\mathbf{f}(\mathbf{r}, \alpha)$ в $\mathbb{R}^{3}$ инвариантно относительно группь вращений куба О или полной группь его симметрий. Тогда

$\{1\}$ в подходящих декартовых координатах уравнения имеют вид

$$
\dot{x}=\lambda(\alpha) x+x\left[a(\alpha) x^{2}+b(\alpha)\left(y^{2}+z^{2}\right)\right]+O\left(R^{4}\right)
$$

(еще два уравнения получаются ииклической перестановкой $x, y, z)$.

Предположим, что $\lambda(0)=0$ и выполнень следующие условия невырожсденности при $\alpha=0\left(a_{0}=a(0), b_{0}=b(0)\right)$ :

$$
\lambda_{1}=\lambda^{\prime}(0) \neq 0, \quad a_{0} \neq 0, \quad\left|a_{0}\right| \neq\left|b_{0}\right|, \quad a_{0}+2 b_{0} \neq 0 .
$$

Тогда при достаточно мальх $\alpha\left(|\alpha|<\varepsilon_{*}\right)$ существует иар $B\left(R \leqslant R_{*}\right)$ со следующими свойствами:

$\{2\}$ для фиксированного $\alpha$ на каждой из осей $s_{k}$ симметрии куба в имеется не более двух положсений равновесия системы, отличных от $\mathbf{r}=\mathbf{0}$ (на данной оси таких равновесий два или ни одного);

$\{3\}$ каждая полутраектория системь, начинающаяся в $B$, с ростом $t$ стремится к одному из указанных положсений равновесия или покидает иар В за конечное время. 
ДоказАтельство. 1) Справедливость утверждения $\{1\}$ следует из п. 5.1.

2) На каждой из осей симметрии система сводится к уравнению вида $\dot{s}=\lambda_{1} \alpha s+$ $A s^{3}+O\left(\alpha^{2}|s|+\left|\alpha s^{3}\right|+s^{4}\right)$. В силу условий (5.1) коэффициенты $A$ для всех осей отличны от нуля. Правая часть уравнения обрашается в нуль при $s=0$ и при $s= \pm\left(-\lambda_{1} \alpha / A\right)^{1 / 2}+O(\alpha)$ (для $\alpha>0$ или для $\left.\alpha<0\right)$. Все эти уравнения не имеют других положений равновесий при $|\alpha|<\varepsilon_{1}$ и $R<R_{1}$.

3) Основное утверждение $\{3\}$ выводится из леммы 3.1 точно так же, как в теореме 4.1 .

\section{§6. Симметрия додекаэдра}

В этом параграфе используются сведения (и терминология) из дополнения.

6.1. Об инвариантах и эквивариантах. Пусть $Y \subset \mathrm{SO}(3)$ - группа вращений додекаэдра. Из общей теоремы об инвариантах этой групшы вытекают следующие утверждения об однородных инвариантах $I_{n}$ невысокой степени $n:$ a) $I_{2}=$ $c R^{2}$; б) $I_{4}=c R^{4}$; в) существует нетривиальный (т.е. зависящий не только от $R$ ) инвариант шестой степени $P_{6}$, и произвольный инвариант $I_{6}$ равен $a P_{6}+c R^{6}$. Добавим к этому, что не существует ненулевых инвариантов степеней 3 и 5 . (Элементарные доказательства этих утверждений приведены в дополнении.)

$Y$-эквивариантное векторное поле, компоненты которого - многочлены степени не вьше 6, обязательно градиентно. Таким образом, если ограничиться тейлоровскими слагаемыми не выше пятой степени, то система дифференциальных уравнений в $\mathbb{R}^{3}$, инвариантная относительно группы $Y$, имеет вид

$$
\dot{\mathbf{r}}=\lambda \mathbf{r}+\mathbf{r}\left(a_{2} R^{2}+a_{4} R^{4}\right)+a \operatorname{grad} P_{6}(\mathbf{r})
$$

6.2. Нетривиальный инвариант шестой степени. Напомним, что додекаэдр имеет центр симметрии и следующие оси вращательной симметрии:

1) 6 осей пятого порядка. Каждая такая прямая ортогональна двум противоположным граням в их центрах.

2) 10 осей третьего порядка, соединяющих пары противоположных вершин.

3) 15 осей второго порядка, соединяющих середины противолежащих ребер.

Группа $Y$ содержит 60 элементов и действует транзитивно на множестве осей каждого типа.

Пусть уравнения $l_{k}(\mathbf{r})=0(k=1, \ldots, 6)$ задают плоскости, ортогональные осям пятого порядка и проходящие через точку $\mathbf{r}=\mathbf{0}$. Пусть линейные формы $l_{k}$ нормированы: сумма квадратов коэффициентов равна 1 . Положим $P_{6}(\mathbf{r})=\prod l_{k}(\mathbf{r})$. Полученный многочлен (определенный с точностью до знака) инвариантен относительно действия элементов $g$ группы $Y$. Действительно, $l_{k}(g \mathbf{r})= \pm l_{j}(\mathbf{r})$ (разным $k$ отвечают разные $j)$ и поэтому a priori $P_{6}(g \mathbf{r})= \pm P_{6}(\mathbf{r})$, но для элементов $g$ пятого порядка знак "минус" невозможен, а наименьшая подгруппа, содержашая все такие элементы, совпадает со всей группой.

6.3. Угловая система. Пусть снова $\Omega$ пробегает единичную сферу $(\Omega=\mathbf{r} / R)$ и $d \tau=R^{4} d t$. Следствием системы (6.1) является градиентная система на сфере $\Omega^{\prime}=\Phi(\Omega)$, порождаемая функцией $J(\Omega)=a P_{6} / R^{6}$ (штрих заменяет $d / d t$ ). Положениям равновесия $\Omega_{k}$ этой системы отвечают лучи, инвариантные для (6.1). 
Можно показать, что при $a \neq 0$ имеется лишш конечное число точек $\Omega_{k}$ (см. дополнение).

Лемма 6.1. При $a \neq 0$ 1) система $\Omega^{\prime}=\Phi(\Omega)$ имеет в качестве положений равновесия только точки, отвечающие осям вращения $s_{j}$ группы $Y$; 2) все положения равновесия әтой системы гиперболические.

ДокАЗАтельство. 1) Введем в $\mathbb{R}^{3}$ координаты $(x, y, z)$ так, чтобы в плоскости $x=0$ не было инвариантных лучей системы (6.1) (определяемых последним слагаемым правой части). На инвариантных лучах должны выполняться уравнения (индекс 6 опушен):

$$
y P_{x}^{\prime}-x P_{y}^{\prime}=0, \quad z P_{y}^{\prime}-y P_{z}^{\prime}=0, \quad x P_{z}^{\prime}-z P_{x}^{\prime}=0 .
$$

Рассмотрим в полупространстве $x>0$ первые два уравнения (6.2). Положив $\eta=y / x$ и $\zeta=z / x$ (и поделив на $x^{6}$ ), получим систему П двух уравнений шестой степени: $\Pi_{1}(\eta, \zeta)=0, \Pi_{2}(\eta, \zeta)=0$. Система П имеет (вешественные) решения, отвечающие осям $s_{j}$; таких решений 31 . Предположим, что есть инвариантная прямая $s$ системы (6.1), отличная от всех осей $s_{j}$. Под действием преобразований группы $Y$ из $s$ получится 59 (или 29) других прямых, также инвариантных для (6.1). Но система П не может иметь более 36 решений и, следовательно, имеет их ровно 31 . Тем самым, система $\Omega^{\prime}=\Phi(\Omega)$ имеет ровно 62 положения равновесия (для $x>0$ и для $x<0$ ).

2) Предположим, что одна из точек $\Omega_{k}$ - вырожденное положение равновесия системы $\Omega^{\prime}=\Phi(\Omega)$. Тогда будут вырожденными не менее 12 равновесий $(12-$ минимальное число точек в орбите группы $Y$ ). Итак, при сделанном предположении система П имеет $m \geqslant 6$ вырожденных решений и $31-m$ невырожденных. Алгебраическая кратность вырожденных решений не менее 2, а полное число решений с учетом кратности не может быть более 36 в силу теоремы Безу [10; гл. 4: раздел 1.1, пример 3 и раздел 2.1, пример 1]. Это при $m \geqslant 6$ невозможно.

6.4. ПРЕДЛОЖЕНИЕ 6.2. Пусть семейство дифференциальных уравнений $\dot{\mathbf{r}}=\mathbf{f}(\mathbf{r}, \alpha)$ в $\mathbb{R}^{3}$ инвариантно относительно группь вращений додекаәдра $Y$ или полной группь его симметрий. Тогда

$\{1\}$ уравнения имеют вид

$$
\dot{\mathbf{r}}=\lambda(\alpha) \mathbf{r}+\mathbf{r} A(R, \alpha)+a(\alpha) \operatorname{grad} P_{6}(\mathbf{r})+O\left(R^{6}\right) ;
$$

здесь $R=|\mathbf{r}|, P_{6}-$ описанный выцее инвариант группы $Y$ и

$$
A(R, \alpha)=a_{2}(\alpha) R^{2}+a_{4}(\alpha) R^{4} .
$$

Предположим, что $\lambda(0)=0$ и выполнены следующие условия невырожденности при $\alpha=0$ :

$$
\lambda_{1}=\lambda^{\prime}(0) \neq 0, \quad a_{2}=a_{2}(0) \neq 0, \quad a=a(0) \neq 0 .
$$


Тогда при достаточно мальх $\alpha\left(|\alpha|<\varepsilon_{*}\right)$ существует иар $B\left(R \leqslant R_{*}\right)$ со следующими свойствами:

$\{2\}$ для фиксированного а на каждой из 31 вращательньх осей $s_{k}$ группьи $Y$ в В имеется не более двух положений равновесия системы, отличных от $\mathbf{r}=\mathbf{0}$. Других равновесий в В система не имеет;

$\{3\}$ каждая полутраектория системь, начинающаяся в $B$, с ростом $t$ стремится к одному из положсений равновесия либо покидает шар $B$ за конечное время.

Уточнения и комментарии. а) $\operatorname{Ecли~} \operatorname{sign}(\alpha)=\operatorname{sign}\left(\lambda_{1} a_{2}\right)$, то в $B$ нет никаких равновесий, кроме $\mathbf{r}=\mathbf{0}$. Доказательство очевидно: в этом случае $\dot{R}$ сохраняет знак при $R<R_{*}$.

б) Если $\operatorname{sign}(\alpha)=-\operatorname{sign}\left(\lambda_{1} a_{2}\right)$, то на каждой из прямых $s_{k}$ имеется ровно два положения равновесия, удаленных от $\mathbf{r}=\mathbf{0}$ на равное расстояние (порядка $|\alpha|^{1 / 2}$ ). Всего, таким образом, при этой бифуркации рождается 62 нетривиальных равновесия, разделенных на три семейства - по 12, 20 и 30 точек. Равновесия каждого семейства переводятся друг в друга преобразованиями группы и “устроены одинаково".

в) Условия $a_{2}(0) \neq 0$ и $a(0) \neq 0$ можно заменить словесной формулировкой: при $\alpha=0$ в системе должны иметься ненулевые кубичные слагаемые, а члены пятой степени не должны обладать сферической симметрией.

Доказательство предложения 6.2 аналогично доказательству теоремы 4.1. Оно основано на лемме 3.1 и сведениях об угловой системе, приведенных в п. 6.3.

\section{§7. Симметрия $T_{h}$}

В этом параграфе кратко рассмотрен случай, в котором модельная система не градиентна, а в роли аттрактора может выступать цикл, образованный тремя гетероклиническими траекториями.

7.1. Вид уравнений. Пусть система уравнений $\dot{\mathbf{r}}=\mathbf{f}(\mathbf{r})$ в $\mathbb{R}^{3}(\cdot=d / d t)$ инвариантна относительно групшы $T_{h}$, порожденной группой вращений тетраэдра $T$ и центральной симметрией. Выберем декартовы координаты так, как в $\S 2$. Отражения в координатных плоскостях входят в группу $T_{h}$; в частности, эти плоскости инвариантны для системы. В силу этого $\dot{x}=x \widetilde{u}(x, y, z)$, где $\widetilde{u}$ есть гладкая функция от $x^{2}, y^{2}$ и $z^{2}$; аналогично для $\dot{y}$ и $\dot{z}$. Лемма 2.1 остается в силе. Выписав явно линейные и кубичные члены, получим уравнения

$$
\begin{aligned}
& \dot{x}=x\left[\lambda+a x^{2}+b y^{2}+c z^{2}+O\left(R^{4}\right)\right], \\
& \dot{y}=y\left[\lambda+a y^{2}+b z^{2}+c x^{2}+O\left(R^{4}\right)\right], \\
& \dot{z}=z\left[\lambda+a z^{2}+b x^{2}+c y^{2}+O\left(R^{4}\right)\right] .
\end{aligned}
$$

Чтобы изучить поведение решений (7.1), достаточно рассмотреть "положительный” октант $(x, y, z \geqslant 0)$. Двумерные системы на его гранях устроены одинаково, поскольку прямая $x=y=z$ - ось симметрии третьего порядка. 
7.2. Функция Ляпунова. Отбросив в (7.1) остаточные члены, получим

$$
\dot{x}=x\left(\lambda+a x^{2}+b y^{2}+c z^{2}\right) \text { и т. д. (по циклу). }
$$

При $b \neq c$ система (7.2) не градиентна. Введем, как в п. 2.2, “угловую” систему $\Omega^{\prime}=\Phi(\Omega)$ (производная по $\left.\tau, d \tau=R^{2} d t\right)$ ). Она тоже не градиентна, но допускает “функцию Ляпунова” $J(\Omega)=x y z / R^{3}$. Производная $J$ в силу угловой системы $J_{d}$ дается формулами:

$$
\begin{gathered}
J_{d}(\Omega)=K J(\Omega) F(\Omega), \quad K=b+c-2 a, \\
F(\Omega)=\left(x^{4}+y^{4}+z^{4}-x^{2} y^{2}-y^{2} z^{2}-z^{2} x^{2}\right) / R^{4} .
\end{gathered}
$$

Функция $F$ неотрицательна и в сферическом треугольнике $\Delta$, отвечающем выбранному октанту, обрашается в нуль (имея невырожденный минимум) лишњ в точке $\Omega_{0}$, где $x=y=z$. Пусть $K \neq 0$. Тогда $\operatorname{sign} J_{d}=\operatorname{sign} K$ в треугольнике $\Delta$ везде, кроме его центра $\Omega_{0}$ и границы (где $\left.J \equiv 0\right)$. Рассмотрим теперь $J(\Omega)(\Omega=\mathbf{r} / R)$ как (однородную) функцию $\mathbf{r}$.

ЛЕмма 7.1. Функиия $\Lambda(x, y, z)=x y z / R^{3}$ при $K \neq 0$ является в положительном октанте функиией Ляпунова системы (7.1) в следующем смысле. Пусть $\mathbf{r}(t)$ - решение этой системь. Существует $R_{1}$ такое, что при $|\mathbf{r}(t)|<R_{1}$ функиия $V(t)=\Lambda(\mathbf{r}(t))$ меняется монотонно. При этом производная $\dot{V}(t)$ обращается в нуль, только если траектория $\mathbf{r}(t)$ лежит на луче $l$ $(x=y=z)$ или на одной из координатных плоскостей.

Доказательство похоже на приведенное в $\S 3$.

СЛЕДСТВИЕ 7.2. Полутраектория системы (7.1) в положсительном октанте, для которой $R(t)<R_{1}$, с ростом $t$ стремится к лучу $l$ илик поверхности октанта. Более точно: если $K<0$, то любая полутраектория, не лежащая на l, стремится к обвединению граней октанта. При $K>0$ любая полутраектория, не лежащая на одной из граней, с ростом $t$ стремится $\kappa$ лучy $l$.

7.3. Двумерные системы. На грани $z=0$ получим, отбросив $O\left(R^{4}\right)$ :

$$
\dot{x}=x\left(\lambda+a x^{2}+b y^{2}\right), \quad \dot{y}=y\left(\lambda+a y^{2}+c x^{2}\right)
$$

Эта система имеет (в квадранте $x \geqslant 0, y \geqslant 0$ ) нетривиальные положения равновесия на координатных осях при $\lambda a<0$. Равновесия гиперболичны, если выполнены неравенства: $A=a-b \neq 0$ и $C=c-a \neq 0$. Если $A C>0$, то других равновесий нет и имеется единственная траектория, соединяющая эти два. При $A C<0$ система (7.3) имеет инвариантный луч внутри квадранта, и на нем может быть еще одно положение равновесия (не подсказьваемое симметрией). Уравнения (7.3) не имеют замкнутых траекторий: введя переменные $\xi=\ln x$ и $\eta=\ln y$, получим систему, у которой дивергенция правой части сохраняет знак. Легко проверить также, что система (7.3) не имеет сепаратрисных циклов.

В нашей бифуркационной задаче $\lambda(\alpha)=\lambda_{1} \alpha+O\left(\alpha^{2}\right)$. Если $\lambda_{1} \neq 0$, то сделанные утверждения о системах на гранях справедливы для (7.1) в области $R \leqslant R_{2}$ при $0<|\alpha|<\varepsilon_{*}$. 
7.4. ПРЕДЛОЖЕНИЕ 7.3. Пусть семейство дифференциальных уравнений $\dot{\mathbf{r}}=\mathbf{f}(\mathbf{r}, \alpha)$ в $\mathbb{R}^{3}$ инвариантно относительно группь $T_{h}$. Тогда в подходящих декартовых координатах уравнения имеют вид (7.1), где коэффициенты зависят от $\alpha$.

Пусть $\lambda(0)=0$ и при $\alpha=0$ выполнень следующие неравенства:

$$
\lambda_{1}=\lambda^{\prime}(0) \neq 0, \quad a \neq 0, \quad a \neq b, \quad a \neq c, \quad b+c \neq 2 a
$$

Тогда при достаточно мальх $\alpha\left(|\alpha|<\varepsilon_{*}\right)$ существует иар $B\left(R \leqslant R_{*}\right)$, обладающий следующим свойством. Каждая траектория r $(t)$ системы, остающаяся в $B$ при всех $t \geqslant 0$, с ростом $t$ стремится $к$ одному из положсений равновесия или $\kappa$ гетероклиническому ииклу, образованному из трех равновесий и соединяющих их траекторий.

ЗАмечАния. 1) Речь идет, кроме $\mathbf{r}=\mathbf{0}$, о равновесиях, рождающихся в результате бифуркации - лежащих на осях или плоскостях симметрии (и расположенных на расстояниях порядка $|\alpha|^{1 / 2}$ от $\left.\mathbf{r}=\mathbf{0}\right)$.

2) Пусть снова $A=a-b, C=c-a, K=b+c-2 a$. Гетероклинические циклы содержат седловые равновесия, лежащие на трех координатных осях. Они существуют при $A C>0$ (и подходящем знаке $\alpha$ ). При $K<0$ и отталкивающем равновесии $\mathbf{r}=\mathbf{0}\left(\lambda_{1} \alpha>0, a<0\right)$ такой цикл притягивает к себе все траектории в шаре $B$ из положительного координатного октанта, кроме особых - тех, для которых $x=y=z$ или одна из координат тождественно равна нулю.

3) Основная идея доказательства предложения 7.3 - использование “функции Ляпунова" $\Lambda$ (п. 7.2). Затем нужно рассмотреть, каким может быть $\omega$-предельное множество $M$ “внутренней” траектории, если оно расположено на гранях положительного октанта. В силу сказанного вьше $M$ может состоять только из равновесий и соединяющих их траекторий. Доказывается, что из всех мыслимых комбинаций реализуются только две: $M$ есть равновесие или указанный гетероклинический цикл.

4) Система (7.1) рассмотрена также в работе [11]. Основная цель авторов этой статьи - показать, что в симметричных системах гетероклинический цикл может быть “структурно устойчив". Предельное поведение траекторий описано там для случая, когда гетероклинический цикл является "главньп аттрактором" (см. замечание 2) выше).

\section{Дополнение: инварианты и эквиварианты}

Д.1. Пусть $G$ - подгруппа $\mathrm{SO}(3)$. Выберем декартову систему координат и рассмотрим инварианты группы $G$ - многочлены $P(x, y, z)$, для которых $P(g \mathbf{r})=$ $P(\mathbf{r})$ при всех $g \in G$. Всегда есть тривиальные инварианты вида $Q\left(R^{2}\right)$, пригодные для всех подгрупп $\mathrm{O}(3)\left(R^{2}=x^{2}+y^{2}+z^{2}\right)$; остальные инварианты $G$ будем называть нетривиальньми.

Если $P$ - инвариант $G$, то $\mathbf{f}=\operatorname{grad} P$ - векторное поле, зквивариантное относительно действия $G$. Назовем зквивариантные поля вида $\mathbf{f}(\mathbf{r})=\mathbf{r} a(R)=$ $\operatorname{grad} Q\left(R^{2}\right)$ тривиальньми. Будем обозначать через $\mathbf{f}_{n}$ векторные поля, компоненты которых - однородные многочлены степени $n$. 
Д.2 ЛЕмма Д.2. Пусть $\mathbf{f}_{n}(\mathbf{r})$ - нетривиальный әквивариант группъ $G$, имеющий наименьшую степень $n$. Тогда векторное поле $\mathbf{f}_{n}$ градиентно.

ДоКАЗАТЕЛЬСТВо. Положим $\mathbf{F}_{n-1}(\mathbf{r})=\operatorname{rot} \mathbf{f}_{n}(\mathbf{r})$. Поле $\mathbf{F}_{n-1}$ эквивариантно относительно $G$ и, значит, по выбору $n \mathbf{F}_{n-1}=\mathbf{r} a(R)$. Поскольку $\operatorname{div} \mathbf{F}_{n-1} \equiv 0$, то $a(R) \equiv 0$. Итак, $\mathbf{f}_{n}=\operatorname{grad} P_{n+1}$. Очевидно, $P_{n+1}(\mathbf{r})-$ нетривиальньй инвариант групшы $G$.

Д.3. Пусть $\Gamma$ - конечная группа ортогональных преобразований $\mathbb{R}^{n}$, порожденная отражениями. Тогда всякое полиномиальное векторное поле $\mathbf{f}$, эквивариантное относительно Г, или градиентно, или есть линейная комбинация градиентных полей: $\mathbf{f}=\sum_{k=1}^{k=n} a^{(k)} \operatorname{grad} I^{(k)}$. Здесь $a^{(k)}$ и $I^{(k)}$ - инвариантные многочлены $\left(I^{(k)}\right.$ образуют базис в алгебре инвариантных многочленов). Это утверждение - частньй случай теоремы, доказанной в [12] (см. также [13; с. 169]).

В нашем случае $I^{(1)}=R^{2}$, а нетривиальные инварианты $I^{(2)}$ имеют следуюшие степени: 3 - для полной групшы $T_{d}$ симметрий тетраэдра, 4 - для группы $O_{h}$ симметрий куба и 6 - для группы $Y_{h}$ всех симметрий додекаэдра. Степени нетривиальных инвариантных многочленов $a^{(k)}$ не менее 3,4 и 6 соответственно. Поэтому полиномиальные векторные поля, эквивариантные относительно этих групп, обязательно градиентны, если их степень не превосходит: 3 для групшы $T_{d}, 4$ для группы $O_{h}$ и 6 для $Y_{h}$.

Д.4. Инварианты невысокой степени для группы вращений додекаэдра $Y$. Выберем декартову систему координат так, чтобы одна из граней додекаэдра была параллельна плоскости $x O y$. Ось $z$ будет тогда осью симметрии пятого порядка. Середины 10 ребер и, соответственно, 5 осей симметрии второго порядка будут лежать в плоскости $x O y$. Выберем одну из них в качестве оси $x$. Пусть $I_{n}(x, y, z)$ - однородный многочлен, инвариантный относительно групшы $Y$. Запишем его в виде

$$
I_{n}=c_{0} z^{n}+p_{1}(x, y) z^{n-1}+\cdots+p_{n-1}(x, y) z+p_{n}(x, y)
$$

Многочлены $p_{k}(x, y)$ должны быть инвариантны относительно поворота на угол $2 \pi / 5$. Отсюда

$$
p_{1}=p_{3} \equiv 0, \quad p_{2}=c_{2} r^{2}, \quad p_{4}=c_{4} r^{4}, \quad p_{6}=c_{6} r^{6} \quad\left(r^{2}=x^{2}+y^{2}\right) .
$$

Далее, $p_{5}(x, y)=\operatorname{Re}\left[C(x+i y)^{5}\right]$. Из этих равенств вытекает, что инварианты второй и четвертой степени не изменяются при повороте вокруг оси $z$ на любой угол. Они будут, следовательно, инвариантны относительно поворотов вокруг любой другой оси пятого порядка. Две такие однопараметрические подгрупшы порождают всю группу $\mathrm{SO}(3)$ и, значит, $I_{2}=a_{2} R^{2}, I_{4}=a_{4} R^{4}$. Инварианты третьей и пятой степени должны иметь вид:

$$
I_{3}=c_{0} z^{3}+c_{2} r^{2} z \quad \text { и } I_{5}=c_{0} z^{5}+c_{2} r^{2} z^{3}+c_{4} r^{4} z+p_{5}(x, y) .
$$

При повороте на $\pi$ вокруг оси $x$ эти многочлены не должны изменяться. Отсюда $I_{3} \equiv 0$, а в $I_{5}$ первые три слагаемых отсутствуют. Поскольку есть наклонные оси 
симметрии, $I_{5}$ не может быть функцией только от $x$ и $y$ и, значит, $I_{5} \equiv 0$. Займемся инвариантами шестой степени.

Если $I_{6}$ - инвариант группы $Y$, то $\Delta I_{6}$ также инвариант ( $\Delta$ - оператор Лапласа). Вычитая $C R^{6}$, можно сделать $I_{6}$ гармонической функцией. Запишем такой инвариант в виде

$$
I_{6}^{(0)}=z^{6}+c_{2} r^{2} z^{4}+c_{4} r^{4} z^{2}+c_{6} r^{6}+c q_{5}(x, y) z
$$

Здесь с учетом выбора оси $x: q_{5}(x, y)=5 x^{4} y-10 x^{2} y^{3}+y^{5}$. Потребовав $\Delta I_{6}^{(0)}=0$, находим: $c_{2}=-5, c_{4}=3, c_{6}=-\frac{1}{7}$. Для определения $c$ нужно использовать какую-нибудь из наклонных осей симметрии. (В том, что $c$ определится однозначно, можно убедиться без вычислений. Разность двух гармонических инвариантов (Д.1) состоит из одного (последнего) слагаемого. Оно, однако, при $c \neq 0$ не инвариантно относительно группы $Y$, поскольку не инвариантна сумма первых 4 слагаемых.) Произвольньй инвариант шестой степени получается из $I_{6}^{(0)}$ умножением на константу и добавлением $C R^{6}$. Указанный в $\oint 6$ инвариант $P_{6}$ обращается в нуль на плоскости $z=0$. Такой многочлен получится (с точностью до множителя), если из (Д.1) вычесть $c_{6} R^{6}$.

Д.5. Конечность числа решений системы (6.2). Первое из уравнений с учетом (Д.1) приобретет вид $z\left(x^{5}-10 x^{3} y^{2}+5 x y^{4}\right)=0$. Решения системы (6.2) могут, следовательно, лежать в плоскости $z=0$ или в одной из вертикальных плоскостей (угол между соседними равен $\pi / 5$ ). Эти 5 плоскостей переводятся друг в друга преобразованиями группы, и достаточно рассмотреть решения (6.2) в плоскостях $z=0$ и $x=0$. При $z=0$ второе уравнение сводится к равенству $y q_{5}(x, y)=0$, которое выполняется на 5 прямых (осях второго порядка групшы $Y$ ). В плоскости $x=0$ левая часть второго из уравнений (6.2) не обрашается тождественно в нуль (отличен от нуля, например, коэффициент при $y z^{5}$ ). Следовательно, в этой плоскости есть не более 6 прямых, на которых выполняются уравнения (6.2); одна из этих прямых - ось $z$. То же верно для 4 других вертикальных плоскостей. Тем самым конечность числа прямых, на которых выполняются уравнения (6.2), установлена. Попутно подсчитано, что этих прямых не более $31: 5$ в горизонтальной плоскости и 26 - в вертикальных (поскольку ось $z$ входит в каждую из них).

\section{Список литературы}

1. Шноль Э. Э., Николаев Е.В. О бифуркациях симметричных положений равновесия, отвечающих двукратньм собственньм значениям // Матем. сб. 1999. Т. 190. №9. C. $127-150$.

2. Арнольд В.И., Афраймович В.С., Ильяшенко Ю.С., Шильников Л.П. Теория бифуркаций // Итоги науки и техники. Совр. пробл. матем. Фундам. напр. Динамические системы. Т. 5. М.: ВИНИТИ, 1986. С. 5-218.

3. Арнольд В.И. Дополнительные главы теории обькновенных диффференциальных уравнений. М.: Наука, 1978.

4. Swift J., Barany E. Chaos in the Hopf bifurcation with tetrahedral symmetry: convection in a rotating fluid low Prandtl number // European J. Mech. B Fluids. 1991. V. 10. № 2, suppl. P. 99-104.

5. Guckenheimer J., Worfolk P. Instant chaos // Nonlinearity. 1992. V. 5. P. 1211-1222.

6. Golubitsky M., Stewart I., Schaeffer D. Singularities and groups in bifurcation theory. V. II. New York: Springer-Verlag, 1988. 
7. Шафаревич И. Р. Основные понятия алгебры // Совр. пробл. матем. Фундам. напр. Т. 11. М.: ВИНИТИ, 1986.

8. Ландау Л.Д., Лифиии Е. М. Квантовая механика. Нерелятивистская теория. М.: Наука, 1989.

9. Ruelle D. Bifurcations in the presence of a symmetry group // Arch. Rational Mech. Anal. 1973. V. 51. P. 136-152.

10. Шафаревич И. Р. Основы алгебраической геометрии. Т. 1. М.: Наука, 1988.

11. Guckenheimer J., Holmes Ph. Structurally stable heteroclinic cycles // Math. Proc. Cambridge Philos. Soc. 1988. V. 103. P. 189-192.

12. Solomon L. Invariants of finite reflection groups // Nagoya Math. J. 1963. V. 22. P. 57-64.

13. Бурбаки Н. Группы и алгебры Ли. Гл. IV-VI. М.: Мир, 1972.

Институт математических проблем биологии РАН, Пущино

Поступила в редакцию 10.08.1999 